$\underline{\text { Review Article }}$

\title{
MINI-REVIEW OF PHYTOCHEMICALS OF TEN FICUS SPECIES
}

MUSTAFA MUDHAFAR*, HA ALSAILAWI,M. M. ABDULRASOOL, RUAA K. MOHAMMED JAWAD, MAYS A. D.

Department of Anesthesia Faculty of Altuff Collage-Iraq

Email: almosawy2014@gmail.com

Received: 16 Jun 2021 Revised and Accepted: 31 Aug 2021

ABSTRACT

The present work aimed to review ten species of Ficus which is belong to the Moraceae family.

In total, 36 articles were reviewed by using the online search engine PubMed, Scopus, Google Scholar, and Web of Science.

Ten species of Ficus were searched for phytochemicals documentation, which resulted in 250 secondary metabolites and 400 different compounds. Alkaloids compounds such as liriodenine and lysicamine, terpenes, steroids, and flavonoids were found to have anticancer, antibacterial, antifungal, and other biological activities.

Most Ficus species contain secondary metabolites compounds with different types of application. This mini-review provides insights on Ficus's phytochemical compounds and their importance as medicinal properties.

Keywords: Ficus, Phytochemicals, Moraceae family, Chemical compounds

(C) 2021 The Authors. Published by Innovare Academic Sciences Pvt Ltd. This is an open-access article under the CC BY license (http://creativecommons.org/licenses/by/4.0/) DOI: http://dx.doi.org/10.22159/ijcr.2021v5i4.185. Journal homepage: https://ijcr.info/index.php/journal

\section{INTRODUCTION}

Moraceae is one of the biggest plants family with 37 genus and more than 1100 species [1, 2]. Ficus is a genus from the Moraceae family which has 800 species [3] and about 2000 varieties [4, 5]. Ten species of the Ficus genus (Ficus carica L, Ficus Benjamina, Ficus benghalensis, Ficus religiosa, Ficus deltoidea, Ficus exasperate, Ficus racemosa, Ficus palmata, Ficus hispida, and Ficus capensis) are reviewed based on their phytochemicals and biological activities. About 1000-1200 species were documented on the Moraceae plant. Ficus is one of the genera from Moraceae family [6]. Phytochemical compounds such as alkaloids, terpenes, saponins, flavonoids, and steroids were detected [7,8]. The biological studies had been done on the leaves, stem, bark, root, and fruit [9-11].

Fig. fruit is consumed daily due to its benefits in the diet [12-14]. Many species of ficus have been used as a traditional medicine for many years in the human care system due to their therapeutic properties [15-17]. One of the common ficus species is Ficus carica L [18]. Chemical compounds from various plant parts have been extracted and isolated using different extraction techniques. Dimethyl Sulfoxide, 1,2-diethyl-Cyclooctane, 5(hydroxymethyl)-2-Furancarboxaldehyde, and (1-methylethyl)-Cyclohexane has been isolated from the fruit of $F$. carica [19, 20]; $\alpha$-cadinol, germacrene-D-4-ol, $\gamma$-cadinene, and $\alpha$-muurolene isolated from leaves of $F$. benghalensis [21-23], chlorogenic, p-coumaric, ferulic and syringic acids isolated from $F$. benjamina roots [24-26]; and C-8 glucoside, isoquercitrin-6-O-4-hydroxybenzoate, and quercetin-3- $O$ - $\beta$-rhamnoside from leaves of F. exasperata [27-29]. Among biological activities identified from different parts of Ficus sp were antibacterial, anticancer [30, 22]; antioxidation [31-33]; and anti-inflammatory [34-36].

\section{Methodology}

In total, 52 articles were reviewed by using the online search engine PubMed, Scopus, Google Scholar, and Web of Science. Ten species of Ficus were searched for phytochemicals to report their biological activities. Data were arranged according to the species. The workflow is represented in fig. 1.

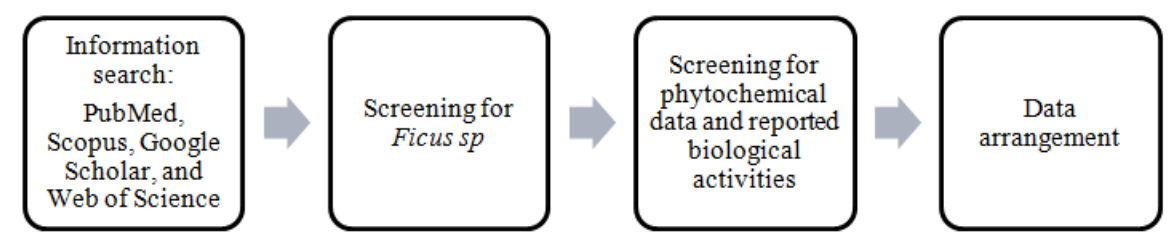

Fig. 1: The workflow

\section{RESULTS AND DISCUSSION}

400 compounds have been shown in 36 articles; these compounds belong to different chemical groups. Table 1 and 2 summarized the compounds identified in Ficus sp. Alkaloids compounds such as liriodenine and lysicamine, terpenes, steroids, and flavonoids were found to have anticancer, antibacterial, antifungal and other biological activities. Most of Ficus species contain secondary metabolites compounds with different types of application. This mini-review provides insights on Ficus's phytochemical compounds and their importance as medicinal properties. 


\begin{tabular}{|c|c|c|}
\hline Spesies & Part & Compounds \\
\hline F. carica $L$ & $\begin{array}{l}\text { Fruit } \\
\text { Latex } \\
\text { Root }\end{array}$ & $\begin{array}{l}\text { Chlorogenic acid(1), Ellagic acid(2), } p \text {-Coumaric acid(3), Kaempferol-rutinoside(4), Quercetin-3-glucoside(5), ferulic } \\
\text { acid(6), 5-O-caffeoylquinic acid (7), Apigenin(8), Catechin(9), Epicatechin(10), Cyanidin-3-glucoside(11), Cyanidin-3- } \\
\text { rutinoside(12) [32, 34,37,38]. }\end{array}$ \\
\hline F. benjamina & $\begin{array}{l}\text { Root, } \\
\text { leaves }\end{array}$ & $\begin{array}{l}\text { 2-Pentanone(13), Hexadecanoic acid(14), Palmitic acid(15), 9,12-Octadecadienoic acid(16), Methanamine(17), } \\
\text { Cyclopentanone(18), Methyl-2 Phenylindole(19), Cyclopropaneoctanal(20), } \\
\text { Arsenous acid(21), (1),(6), Syringic acid(22), Caffeic acid (23) [11, 27, 39]. }\end{array}$ \\
\hline $\begin{array}{l}\text { F. } \\
\text { benghalensis }\end{array}$ & leaves & $\begin{array}{l}\text { Gallic acid(24),Benzoic acids(25), Rhein(26), Theaflavins(27), Anthraquinone(28),Theaflavin-3,3'-digallate (29) [23, } \\
\text { 35]. }\end{array}$ \\
\hline $\begin{array}{l}\text { Ficus } \\
\text { religiosa }\end{array}$ & Stem & $\begin{array}{l}\beta \text {-sitosteryl-D-glucoside (30), n-octacosanol(31), methyl oleanolate(32), stigmasterol(33), lanosterol (34), lupen-3-one } \\
(35)[40,41] .\end{array}$ \\
\hline $\begin{array}{l}\text { Ficus } \\
\text { deltoidea }\end{array}$ & Leaves & $\begin{array}{l}\text { 3b-hydroxy-21aH-Hop22(29)-ene (36), vitexin (37), isovitexin (38),2,4-Bis (dimethylbenzyl)-6-t-butylphenol (39), } \\
\text { Octaethylene glycol (40), Phthalic acid (41), 2-Pentadecanone, 6, 10, 14-trimethyl (42) [42-45]. }\end{array}$ \\
\hline $\begin{array}{l}\text { Ficus } \\
\text { exasperate }\end{array}$ & Leaves & $\begin{array}{l}\alpha \text {-Pinene (43), p-Cymene (44), } \beta \text {-Caryophyllene (45), 6,10,14-Trimethyl-2-pentadecanone (46), 9-Octadecenoic acid } \\
\text { (47), 3-0-glycerolacetate (48), Methyl-2,3-dihydroxyhexacosanoate (49), Oxypeucedanin Hydrate (50) [46-48]. }\end{array}$ \\
\hline $\begin{array}{l}\text { Ficus } \\
\text { racemosa }\end{array}$ & $\begin{array}{l}\text { Leaves } \\
\text { Root }\end{array}$ & Arbutin (51), epicatechin (52), n-Hexacosane (53), Polypodatetraene (54), Glycerol acetate (55) [50, 51]. \\
\hline $\begin{array}{l}\text { Ficus } \\
\text { palmata }\end{array}$ & $\begin{array}{l}\text { Plant } \\
\text { Leaves }\end{array}$ & $\begin{array}{l}\text { Germanicol acetate (56), Psoralene (57), Bergapten (58), Vanillic acid (59), Catechin(60), Genistein (61), } \beta \text {-Sitosterol } \\
\text { (62), Stigmasterol(63) [51,52]. }\end{array}$ \\
\hline Ficus hispida & Fruits & $\begin{array}{l}\text { Alpinumisoflavone (64) 7-hydroxycoumarin (65), psoralen (66), marmesin (67), protocatechuic acid (68), 2-(4- } \\
\text { hydroxy-3-methoxy phenyl)ethyl-D-glucopyranoside (69), (6S,9R)-roseoside (70), murrayaculatine (71), betulinic acid } \\
\text { (72) [53-55] }\end{array}$ \\
\hline Ficus & Leaves & vitamins $B_{12}(73), \alpha$-thujene (74), $\alpha$-pinene (75), camphene (76), $\alpha$-phellandrene (77), p-cymene (78), m-cymene (79), \\
\hline capensis & Seeds & $\begin{array}{l}\text { limonene }(80) \text {, terpinolene }(81) \text {, safranal }(82),(Z) \text {-ocimenone }(83), \text { n-tridecane }(84),, n \text {-tetradecane }(85), \text { cyperene } \\
(86) \text {, isocaryophyllene }(87), \alpha \text {-ionone }(88),(E)-\beta \text {-caryophyllene }(89) \text {, geranylacetone }(90)[56,57] .\end{array}$ \\
\hline
\end{tabular}

Table 2: Compounds properties isolated from Ficus sp. source (athors drow by chemdrwo software)

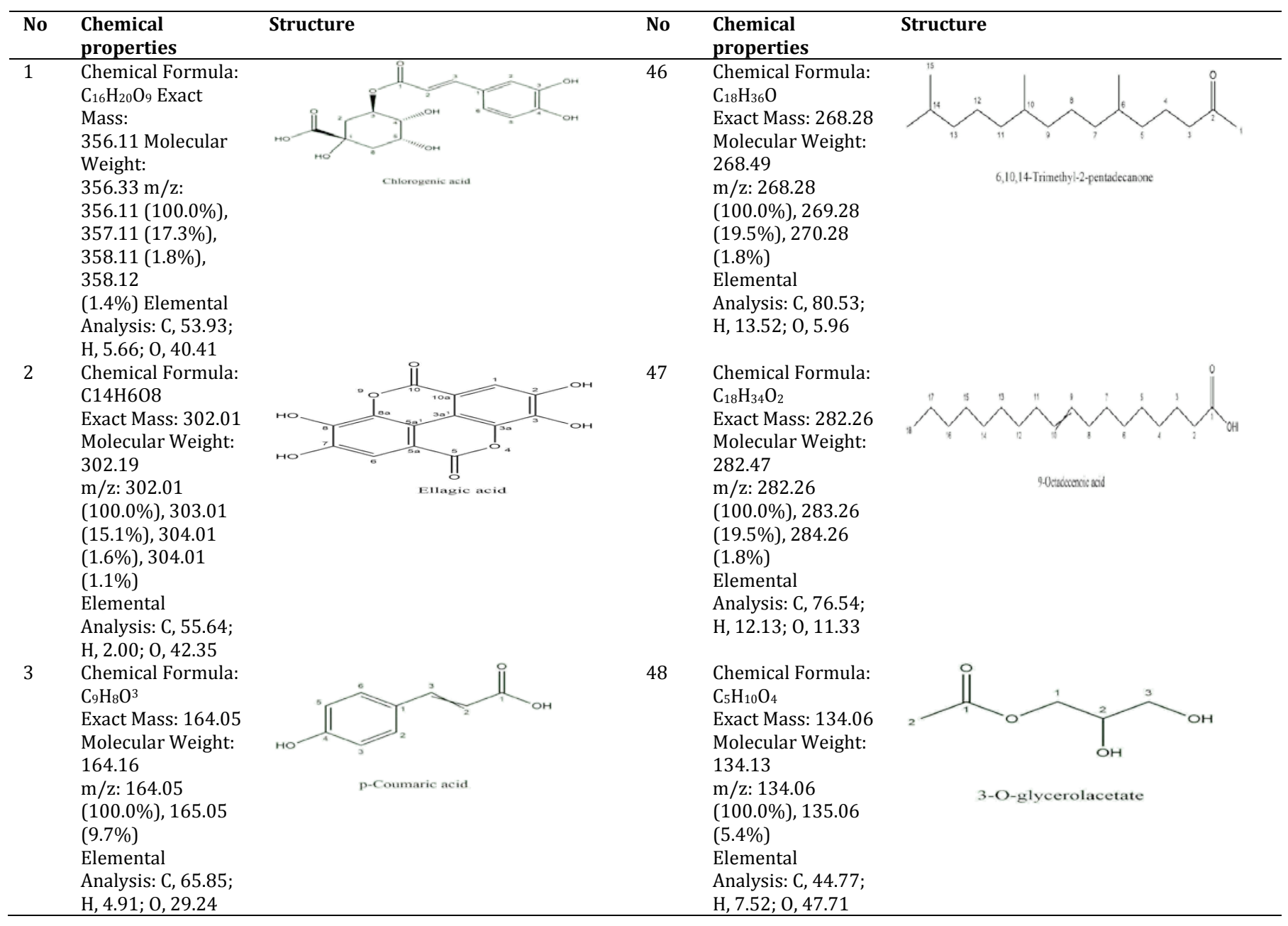




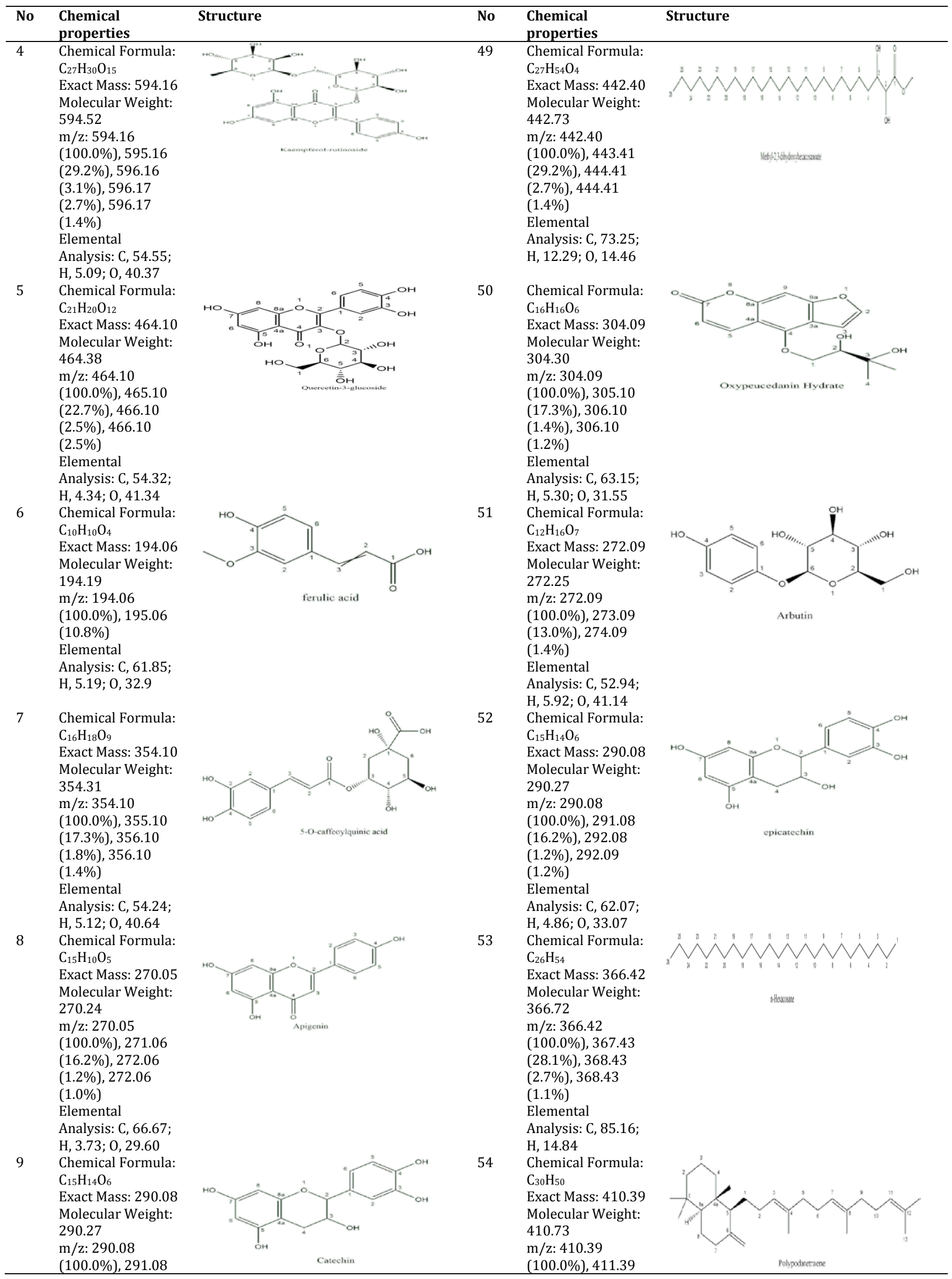




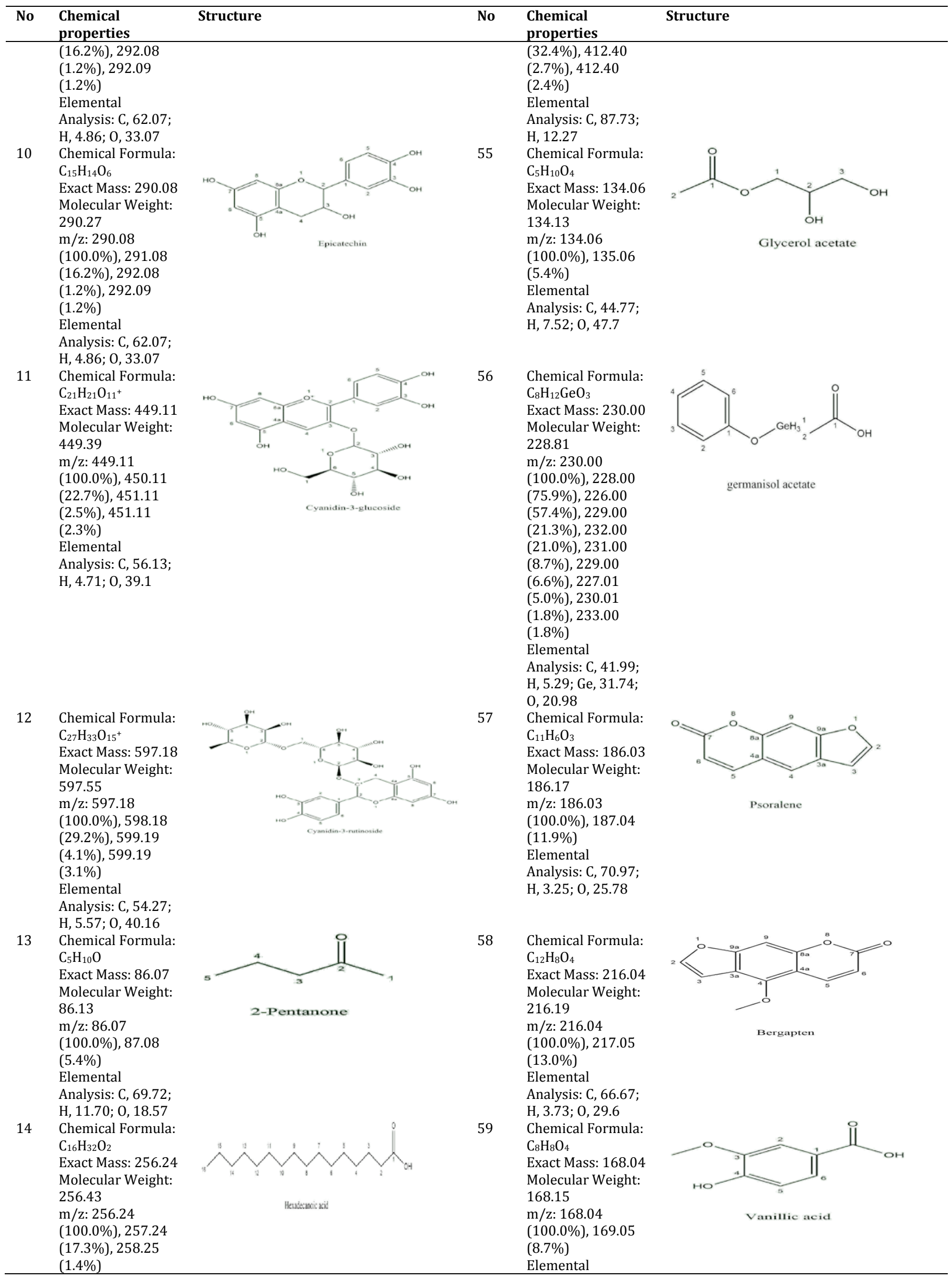




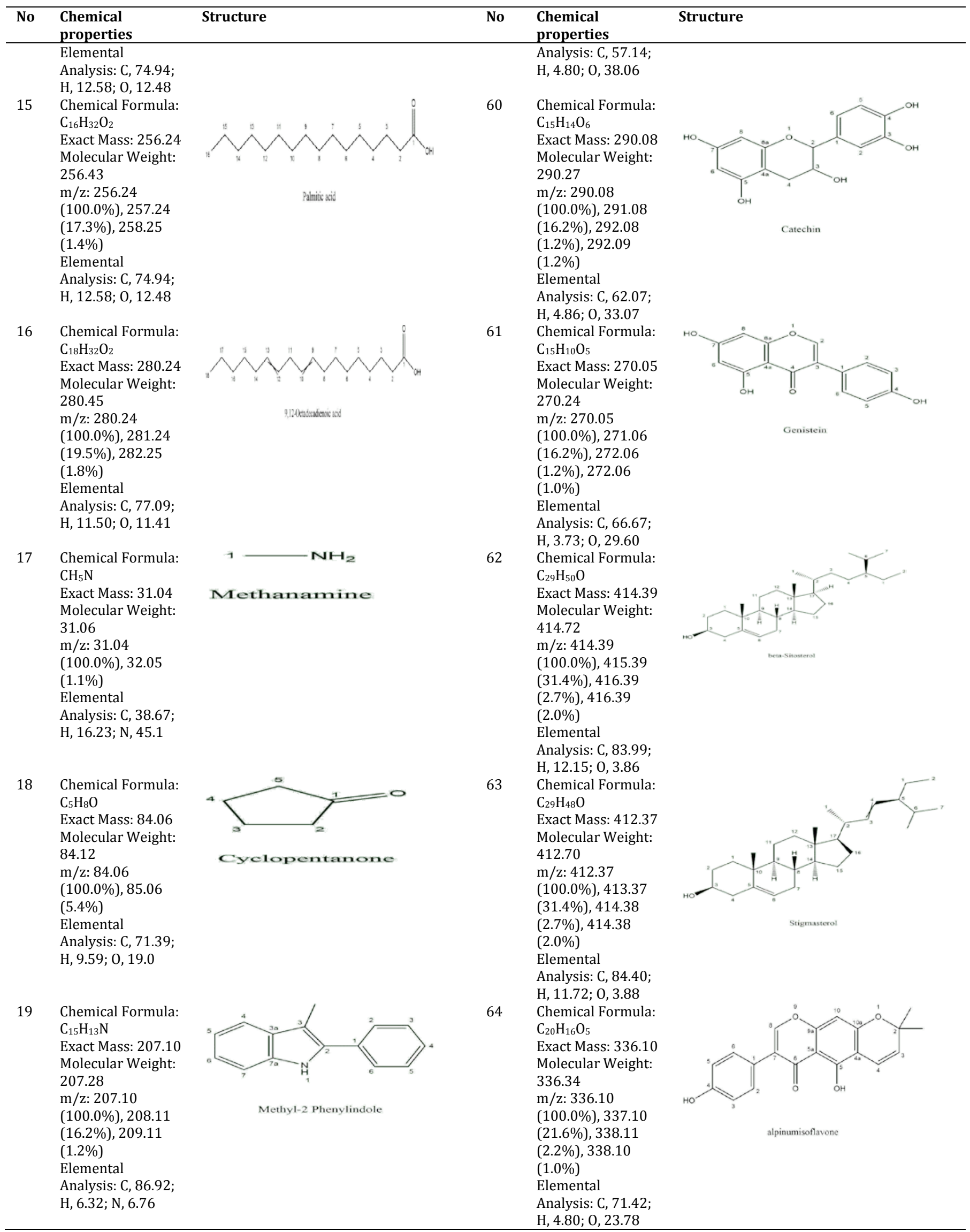




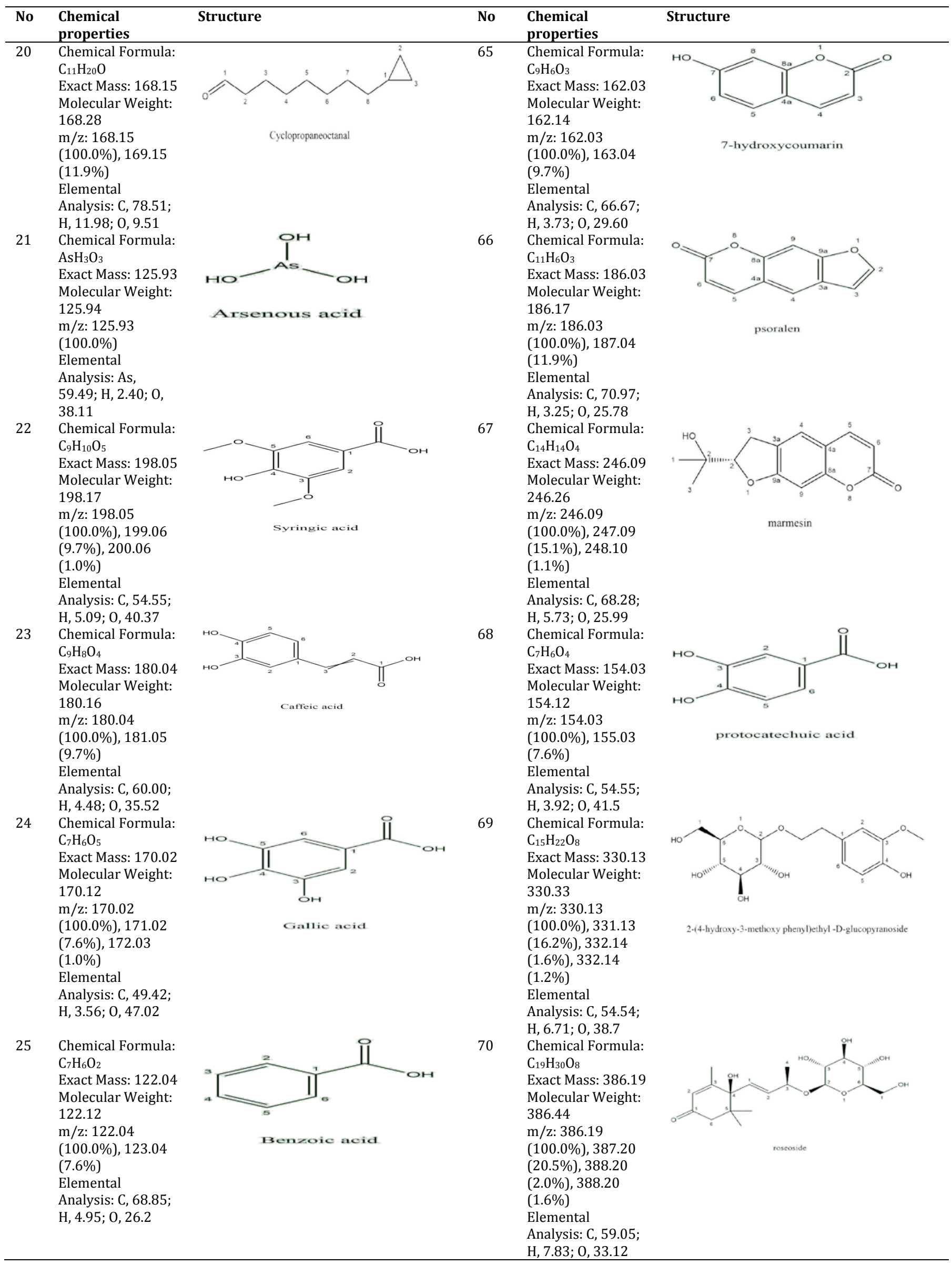




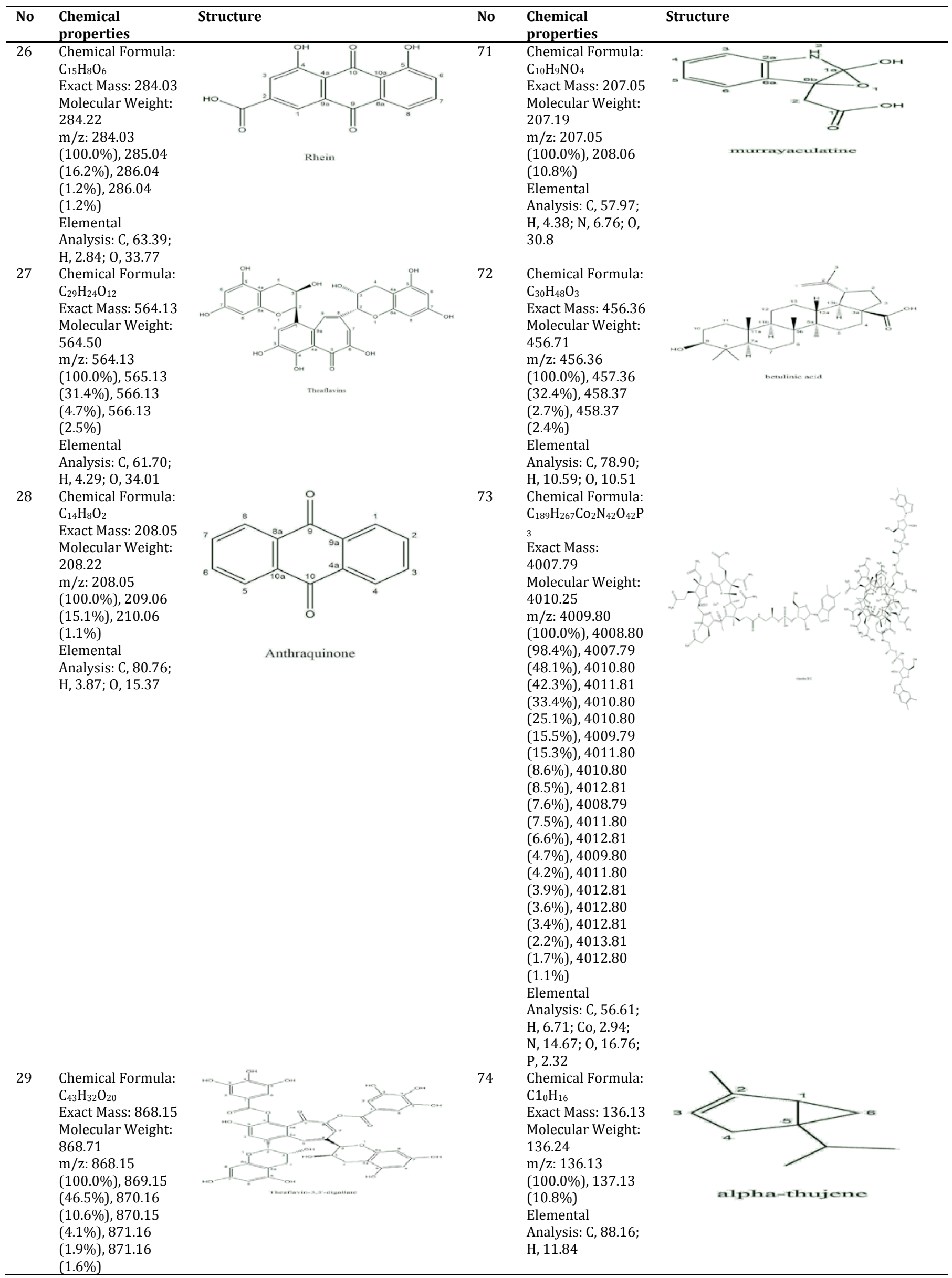




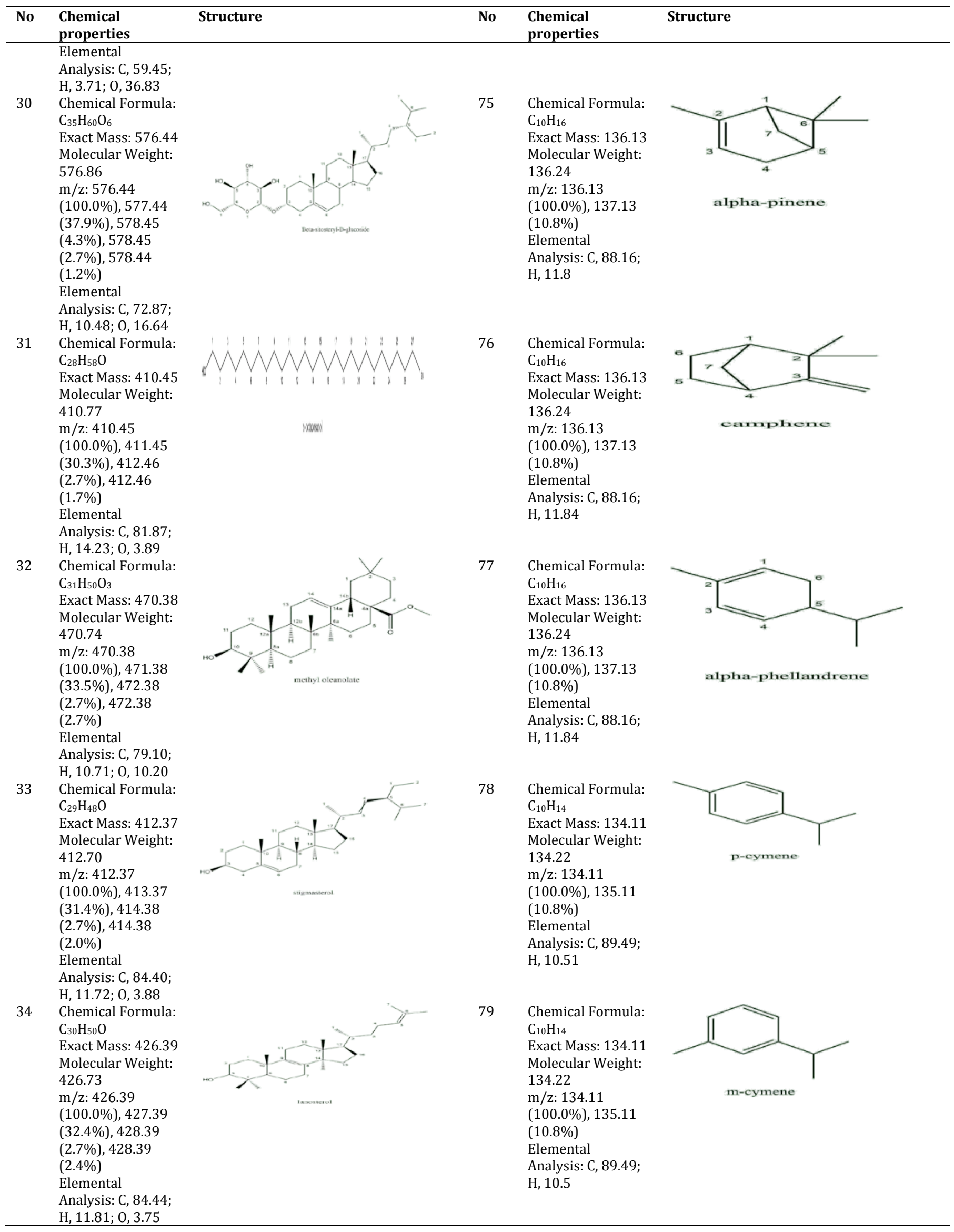




\begin{tabular}{|c|c|c|c|c|c|}
\hline No & $\begin{array}{l}\text { Chemical } \\
\text { properties }\end{array}$ & Structure & No & $\begin{array}{l}\text { Chemical } \\
\text { properties }\end{array}$ & Structure \\
\hline 35 & $\begin{array}{l}\text { Chemical Formula: } \\
\mathrm{C}_{30} \mathrm{H}_{48} \mathrm{O} \\
\text { Exact Mass: } 424.37 \\
\text { Molecular Weight: } \\
424.71 \\
\mathrm{~m} / \mathrm{z}: 424.37 \\
(100.0 \%), 425.37 \\
(32.4 \%), 426.38 \\
(2.7 \%), 426.38 \\
(2.4 \%) \\
\text { Elemental } \\
\text { Analysis: } \mathrm{C}, 84.84 ; \\
\text { H, } 11.39 ; 0,3.7\end{array}$ & & 80 & $\begin{array}{l}\text { Chemical Formula: } \\
\mathrm{C}_{10} \mathrm{H}_{16} \\
\text { Exact Mass: } 136.13 \\
\text { Molecular Weight: } \\
136.24 \\
\mathrm{~m} / \mathrm{z}: 136.13 \\
(100.0 \%), 137.13 \\
(10.8 \%) \\
\text { Elemental } \\
\text { Analysis: } \mathrm{C}, 88.16 ; \\
\mathrm{H}, 11.84\end{array}$ & limonene \\
\hline 36 & $\begin{array}{l}\text { Chemical Formula: } \\
\mathrm{C}_{30} \mathrm{H}_{50} \mathrm{O} \\
\text { Exact Mass: } 426.39 \\
\text { Molecular Weight: } \\
426.73 \\
\mathrm{~m} / \mathrm{z}: 426.39 \\
(100.0 \%), 427.39 \\
(32.4 \%), 428.39 \\
(2.7 \%), 428.39 \\
(2.4 \%) \\
\text { Elemental } \\
\text { Analysis: } \mathrm{C}, 84.44 ; \\
\text { H, } 11.81 ; 0,3.75\end{array}$ & & 81 & $\begin{array}{l}\text { Chemical Formula: } \\
\mathrm{C}_{10} \mathrm{H}_{16} \\
\text { Exact Mass: } 136.13 \\
\text { Molecular Weight: } \\
136.24 \\
\text { m/z: } 136.13 \\
(100.0 \%), 137.13 \\
(10.8 \%) \\
\text { Elemental } \\
\text { Analysis: } \mathrm{C}, 88.16 ; \\
\mathrm{H}, 11.84\end{array}$ & terpinene \\
\hline 37 & $\begin{array}{l}\text { Chemical Formula: } \\
\mathrm{C}_{21} \mathrm{H}_{20} \mathrm{O}_{10} \\
\text { Exact Mass: } 432.11 \\
\text { Molecular Weight: } \\
432.38 \\
\mathrm{~m} / \mathrm{z}: 432.11 \\
(100.0 \%), 433.11 \\
(22.7 \%), 434.11 \\
(2.5 \%), 434.11 \\
(2.1 \%) \\
\text { Elemental } \\
\text { Analysis: } \mathrm{C}, 58.34 ; \\
\text { H, } 4.66 ; 0,37.00\end{array}$ & & 82 & $\begin{array}{l}\text { Chemical Formula: } \\
\mathrm{C}_{10} \mathrm{H}_{14} \mathrm{O} \\
\text { Exact Mass: } 150.10 \\
\text { Molecular Weight: } \\
150.22 \\
\mathrm{~m} / \mathrm{z}: 150.10 \\
(100.0 \%), 151.11 \\
(10.8 \%) \\
\text { Elemental } \\
\text { Analysis: C, } 79.96 \text {; } \\
\text { H, 9.39; 0, } 10.6\end{array}$ & safrenal \\
\hline 38 & $\begin{array}{l}\text { Chemical Formula: } \\
\mathrm{C}_{21} \mathrm{H}_{20} \mathrm{O}_{10} \\
\text { Exact Mass: } 432.11 \\
\text { Molecular Weight: } \\
432.38 \\
\mathrm{~m} / \mathrm{z}: 432.11 \\
(100.0 \%), 433.11 \\
(22.7 \%), 434.11 \\
(2.5 \%), 434.11 \\
(2.1 \%) \\
\text { Elemental } \\
\text { Analysis: } \mathrm{C}, 58.34 ; \\
\text { H, } 4.66 ; 0,37.00\end{array}$ & & 83 & $\begin{array}{l}\text { Chemical Formula: } \\
\mathrm{C}_{10} \mathrm{H}_{14} \mathrm{O} \\
\text { Exact Mass: } 150.10 \\
\text { Molecular Weight: } \\
150.22 \\
\mathrm{~m} / \mathrm{z}: 150.10 \\
(100.0 \%), 151.11 \\
(10.8 \%) \\
\text { Elemental } \\
\text { Analysis: } \mathrm{C}, 79.96 ; \\
\mathrm{H}, 9.39 ; 0,10.6\end{array}$ & (Z)-ocimenon \\
\hline 39 & $\begin{array}{l}\text { Chemical Formula: } \\
\mathrm{C}_{28} \mathrm{H}_{34} \mathrm{O} \\
\text { Exact Mass: } 386.26 \\
\text { Molecular Weight: } \\
386.58 \\
\mathrm{~m} / \mathrm{z}: 386.26 \\
(100.0 \%), 387.26 \\
(30.3 \%), 388.27 \\
(2.7 \%), 388.27 \\
(1.7 \%) \\
\text { Elemental } \\
\text { Analysis: } \mathrm{C}, 87.00 ; \\
\text { H, 8.87; } 0,4.1\end{array}$ & 2,4-Bis (di & 84 & $\begin{array}{l}\text { Chemical Formula: } \\
\mathrm{C}_{13} \mathrm{H}_{28} \\
\text { Exact Mass: } 184.22 \\
\text { Molecular Weight: } \\
184.37 \\
\mathrm{~m} / \mathrm{z}: 184.22 \\
(100.0 \%), 185.22 \\
(14.1 \%) \\
\text { Elemental } \\
\text { Analysis: } \mathrm{C}, 84.69 ; \\
\mathrm{H}, 15.3\end{array}$ & $\overbrace{1}^{8}$ \\
\hline 40 & $\begin{array}{l}\text { Chemical Formula: } \\
\mathrm{C}_{16} \mathrm{H}_{34} \mathrm{O}_{9} \\
\text { Exact Mass: } 370.22 \\
\text { Molecular Weight: } \\
370.44 \\
\mathrm{~m} / \mathrm{z}: 370.22 \\
(100.0 \%), 371.22\end{array}$ & $\bigwedge_{n} V_{i}$ & 85 & $\begin{array}{l}\text { Chemical Formula: } \\
\mathrm{C}_{14} \mathrm{H}_{30} \\
\text { Exact Mass: } 198.23 \\
\text { Molecular Weight: } \\
198.39 \\
\mathrm{~m} / \mathrm{z}: 198.23 \\
(100.0 \%), 199.24\end{array}$ & 1-ietradecane \\
\hline
\end{tabular}




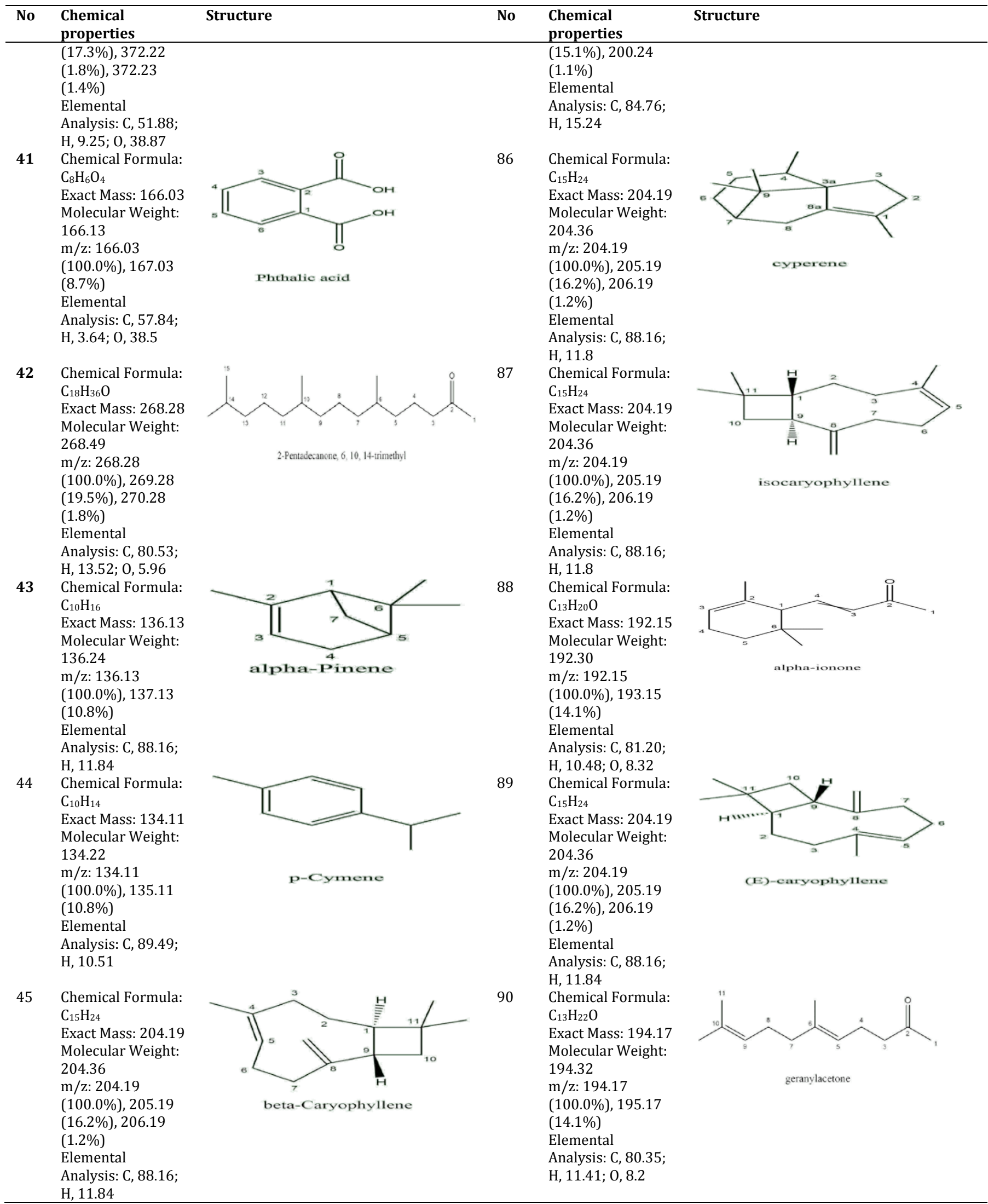

\section{CONCLUSION}

All ten species of Ficus genus have great medicinal values as it has been reported to have phytochemical constituents that is biologically active. In addition to this review will be a good source for future references and database. 


\section{FUNDING}

Nil

\section{AUTHORS CONTRIBUTIONS}

All the authors have contributed equally.

\section{CONFLICT OF INTERESTS}

Declared none

\section{REFERENCES}

1. Burn MJ, Mayle FE. Palynological differentiation between genera of the Moraceae family and implications for Amazonian palaeoecology. Rev Palaeobot Palynol. 2008;149(3-4):187-201. doi: 10.1016/j.revpalbo.2007.12.003.

2. HA Alsailawi, Mustafa Mudhafar, MM Abdulrasool. Effect of frozen storage on the quality of frozen foods- A review. JCCE. 2020;14(3). doi: 10.17265/1934-7375/2020.03.002.

3. Farag MA, Abdelfattah MS, Badr SE, Wessjohann LA. Profiling the chemical content of Ficus lyrata extracts via UPLC-PDA-qTOF-MS and chemometrics. Nat Prod Res. 2014;28(19):1549-56. doi: 10.1080/14786419.2014.926353, PMID 24934244.

4. Salem MZ, Salem AZ, Camacho LM, Ali HM. Antimicrobial activities and phytochemical composition of extracts of Ficus species: an overview. Afr J Microbiol Res. 2013;7:4207-19.

5. Shah SK. Evaluate the antifertility potential of FicusRacemosa linn bark in female Wistar rats. Asian J Pharm Clin Res. 2016;9(6):322. doi: 10.22159/ajpcr.2016.v9i6.14517.

6. Thapliyal MA, Bisht A, Singh AJ. Isolation of antibacterial protein/peptide from Ficus glomerata leaf. Int J Curr Pharm Sci. 2016;8(4):24-7. doi: 10.22159/ijcpr.2016v8i4.15271.

7. Salehi B, Prakash Mishra A, Nigam M, Karazhan N, Shukla I, Kiełtyka Dadasiewicz A, Sawicka B, Głowacka A, Abu Darwish MS, Hussein Tarawneh A, Gadetskaya AV, Cabral C, Salgueiro L, Victoriano M, Martorell M, Docea AO, Abdolshahi A, Calina D, Sharifi Rad J. Ficus plants: state of the art from a phytochemical, pharmacological, and toxicological perspective. Phytother Res. 2021;35(3):1187-217. doi: 10.1002/ptr.6884, PMID 33025667.

8. Chaware GK, Kumar V, Kumar S, Kumar P. Bioactive compounds, pharmacological activity and food application of ficus racemosa: a critical review. Int J Fruit Sci. 2020;20(Suppl2):S969-86. doi: 10.1080/15538362.2020.1774467.

9. Liguori G, Gentile C, Gaglio R, Perrone A, Guarcello R, Francesca N, Fretto S, Inglese P, Settanni L. Effect of addition of opuntia ficus-indica mucilage on the biological leavening, physical, nutritional, antioxidant and sensory aspects of bread. J Biosci Bioeng. 2020;129(2):184-91. doi: 10.1016/j.jbiosc.2019.08.009, PMID 31591026.

10. Ashraf K, Haque MR, Amir M, Ahmad N, Ahmad W, Sultan S, Ali Shah SA, Mahmoud Alafeefy A, Mujeeb M, Bin Shafie MF. An overview of phytochemical and biological activities: Ficus deltoidea Jack and other Ficus spp. J Pharm Bioallied Sci. 2021;13(1):11-25. doi: 10.4103/jpbs.JPBS_232_19, PMID 34084044.

11. Ashraf A, Zafar SA, Ashraf MY, Ijaz MU, Muzammal S, Asad F, Jabeen F, Shahid M. Phytochemical composition and potent biological activities O F Ficus benjamina var. Comosa leaves extract. PAKJBOT. 2020;52(2):531-5. doi: 10.30848/PJB2020-2(3).

12. Pereira C, Lopez Corrales M, Martin A, Villalobos MD, Cordoba MD, Serradilla MJ. Physicochemical and nutritional characterization of brebas for fresh consumption from nine fig varieties (Ficus carica L.) grown in Extremadura (Spain). J Food Qual. 2017;2017:16.

13. Al Sailawi HA, Misnan R, Yadzir ZH, Abdullah N, Bakhtiar F, Arip M, Mudhafar M, Ateshan HM. Effects of different salting and drying methods on the allergenicity of purple mud crab (Scylla tranquebarica). Indian J Ecol. 2020;47:1173-9.

14. Goswami S, Pal N, Singh A, Singh P, Pandey A, Pal R. Fingerprinting of quercetin from Ficus benghalensis. Crit Rev. 2020;13:5699-704.

15. Cheng JX, Zhang BD, Zhu WF, Zhang CF, Qin YM, Abe M, Akihisa T, Liu WY, Feng F, Zhang J. Traditional uses, phytochemistry, and pharmacology of Ficus hispida L.f.: a review. J Ethnopharmacol. 2020;248. doi: 10.1016/j.jep.2019.112204, PMID 112204.

16. Soltana H, Flamini G, Hammami M. Volatile compounds from six varieties of Ficus carica from Tunisia. Rec Nat Prod. 2017;11:157-65.

17. Shahrajabian MH, Sun W, Cheng Q. A review of chemical constituents, traditional and modern pharmacology of fig (Ficus carica L.), a super fruit with astonishing medical characteristics. Pol J Agron. 2021;44:22-9.

18. Wilson VA, Shetye SS, Kaur KA, Shetty SO. Study of synergistic effects on antioxidant activity and antimicrobial activity of polyherbal formulations containing Ficus species. Int J Pharm Pharm Sci. 2016;8:50-3.

19. Shah MD, Yong YS, Iqbal M. Phytochemical investigation and free radical scavenging activities of essential oil, methanol extract and methanol fractions of Nephrolepis biserrata. Int J Pharm Pharm Sci. 2014;6:269-77.

20. Soni N, Mehta S, Satpathy G, Gupta RK. Estimation of nutritional, phytochemical, antioxidant and antibacterial activity of dried fig (Ficus carica). J Pharmacogn Phytochem. 2014;3:158-65.

21. Adebayo MA, Aboaba SA, Eresanya OI, Ajetunmobi AA, Ogunwande IA. Constituent of essential oil from Ficus benghalensis L. Eur J Med Plants. 2015;9(2):1-6. doi: 10.9734/EJMP/2015/18803.

22. Mudhafar M, Zainol I, Jaafar CN, Alsailawi HA, Majhool AA, Alsaady M. Phytochemical screening and characterization of melia dubia leaves extract for antimicrobial activity against escherichia coli and staphylococcus aureus. Indian J Ecol. 2020;47:493-6.

23. Hassan HA, Allam AE, Abu-Baih DH, Mohamed MFA, Abdelmohsen UR, Shimizu K, Desoukey SY, Hayallah AM, Elrehany MA, Mohamed KM, Kamel MS. Isolation and characterization of novel acetylcholinesterase inhibitors from Ficus benghalensis L. leaves. RSC Adv. 2020;10(60):36920-9. doi: 10.1039/D0RA06565J.

24. Imran M, Rasool N, Rizwan K, Zubair M, Riaz M, Zia-Ul-Haq M, Rana UA, Nafady A, Jaafar HZ. Chemical composition and biological studies of Ficus benjamina. Chem Cent J. 2014;8(1):12. doi: 10.1186/1752-153X-8-12, PMID 24524349.

25. Li N, Tu PC, Lo KC, Chang YS. The induction of adventitious roots regeneration before transplanting rootless ficus elastica heritage tree. Forests. 2020;11(10):1057. doi: 10.3390/f11101057.

26. Benedetto AD, Galmarini C, Tognetti J. Differential growth response of green and variegated Ficus benjamina to exogenous cytokinin and shade. Ornam Hortic. 2020;26(2):259-76. doi: 10.1590/2447-536x.v26i2.2089.

27. Taiwo BJ, Igbeneghu OA. Antioxidant and antibacterial activities of flavonoid glycosides from ficus exasperata vahl-holl (Moraceae) leaves. Afr J Tradit Complement Altern Med. 2014;11(3):97-101. doi: 10.4314/ajtcam.v11i3.14, PMID 25371569.

28. Mudhafar M, Zainol I. Medical values, antimicrobial, and antifungal activities of Polyalthia genus. Int J Pharm Res. 2019;11:90-6.

29. Popwo Tameye SC, Djamen Mbeunkeu AB, Fouokeng Y, Jouwa Tameye NS, Tabekoueng GB, Wansi JD, Sewald N, Ndom JC, Azebaze AGB. Ficusanolide $A$ and ficusanolide $B$, two new cinnamic acid derivative stereoisomers and other constituents of the stem barks of ficus exasperata vahl. (Moraceae). Phytochem Lett. 2021;43:150-3. doi: 10.1016/j.phytol.2021.03.027. 
30. Mawa S, Husain K, Jantan I. Ficus carica L. (Moraceae): phytochemistry, traditional uses and biological activities. Evid Based Complement Alternat Med. 2013;2013:974256. doi: 10.1155/2013/974256, PMID 24159359.

31. Kamiloglu S, Capanoglu E. Polyphenol content in figs (Ficus carica L.): effect of sun-drying. Int J Food Prop. 2015;18(3):521-35. doi: 10.1080/10942912.2013.833522.

32. Shahinuzzaman M, Yaakob Z, Anuar FH, Akhtar P, Kadir NHA, Hasan AKM, Sobayel K, Nour M, Sindi H, Amin N, Sopian K, Akhtaruzzaman M. In vitro antioxidant activity of Ficus carica L. latex from 18 different cultivars. Sci Rep. 2020;10(1):10852. doi: 10.1038/s41598-020-67765-1, PMID 32616768.

33. Messina CM, Arena R, Morghese M, Santulli A, Liguori G, Inglese P. Seasonal characterization of nutritional and antioxidant properties of Opuntia ficus-indica [(L.) Mill.] mucilage. Food Hydrocoll. 2021;111. doi: 10.1016/j.foodhyd.2020.106398, PMID 106398.

34. Ali B, Mujeeb M, Aeri V, Mir SR, Faiyazuddin M, Shakeel F. Anti-inflammatory and antioxidant activity of ficus carica linn. leaves. Nat Prod Res. 2012;26(5):460-5. doi: 10.1080/14786419.2010.488236, PMID 21644169.

35. Bhaskara Rao KV, Ojha V, Preeti KG, Kumar G, Karthik L. Phytochemical composition and antioxidant activity of ficus benghalensis (Moraceae) leaf extract. J Biol Act Prod Nat. 2014;4(3):236-48. doi: 10.1080/22311866.2014.936902.

36. Koshak AE, Abdallah HM, Esmat A, Rateb ME. Anti-inflammatory activity and chemical characterization of opuntia ficus-indica seed oil cultivated in Saudi Arabia. Arab J Sci Eng. 2020;45(6):4571-8. doi: 10.1007/s13369-020-04555-x.

37. Jeivad F, Yassa N, Ostad SN, Hassannejad Z, Hassanzadeh Gheshlaghi GH, Sabzevari O. Ficus carica L. latex: possible chemo-preventive, apoptotic activity and safety assessment. Iran J Pharm Res. 2020;19(3):231-40. doi: 10.22037/ijpr.2020.1101151, PMID 33680025.

38. Amani S, Mohebodini M, Khademvatan S, Jafari M, Kumar V. Piriformospora indica based elicitation for overproduction of phenolic compounds by hairy root cultures of Ficus carica. J Biotechnol. 2021;327:43-53. doi: 10.1016/j.jbiotec.2020.12.015, PMID 33387592.

39. Pękala Safińska A, Tkachenko H, Buyun L, Kurhaluk N, Gurnenko I, Honcharenko V, Prokopiv A. Comparative assessment of the antibacterial efficacy of leaf extract obtained from Ficus benjamina L. (Moraceae) and its cultivars against Aeromonas sobria strain. Agrobiodiversity Improv Nutr Health Life Qual. 2021;5:18-25.

40. Chandrasekar SB, Bhanumathy M, Pawar AT, Somasundaram T. Phytopharmacology of ficus religiosa. Pharmacogn Rev. 2010;4(8):195-9. doi: 10.4103/0973-7847.70918, PMID 22228961.

41. Gawande V, Morlock G. Effect-directed profiling of Ficus religiosa leaf extracts for multipotent compounds via 12 effect-directed assays. J Chromatography A. 2021;1637:461836:461836. doi: 10.1016/j.chroma.2020.461836.

42. Lip JM, Hisham DN, Zaidi JA, Musa Y, Ahmad AW, Normah A, Sharizan A. Isolation and identification of moretenol from Ficus deltoidea leaves. J Trop Agric Food Sci. 2009;37:195-201.

43. Abdullah Z, Hussain K, Ismail Z, Ali RM. Anti-inflammatory activity of standardized extracts of leaves of three varieties of Ficus deltoidea. Int J Pharm Clin Res 2009;13:100-5.

44. Choo CY, Sulong NY, Man F, Wong TW. Vitexin and isovitexin from the leaves of ficus deltoidea with in vivo $\alpha$-glucosidase inhibition. J Ethnopharmacol. 2012;1423142(3):776-81. doi: 10.1016/j.jep.2012.05.062, PMID 22683902.

45. Seong Wei, L, Wee W, Yong Fu SiongYong Fu Siong J, Syamsumir DF. Characterization of antioxidant, antimicrobial, anticancer property and chemical composition of Ficus deltoidea Jack. Leaf Extract. leaf extract.J Biol Active Prod Nat.2011;1(1):1-6. doi: 10.1080/22311866.2011.10719067.

46. Sonibare MA, Ogunwande IA, Walker TM, Setzer WN, Soladoye MO, Essien E. Volatile constituents of ficus exasperata leaves. Nat Prod Commun. 2006;1(9). doi: 10.1177/1934578X0600100912.

47. Bafor EE, Lim CV, Rowan EG, Edrada Ebel R. The leaves of ficus exasperata Vahl (Moraceae) generates uterine active chemical constituents. J Ethnopharmacol. 2013;145(3):803-12. doi: 10.1016/j.jep.2012.12.020, PMID 23266275.

48. Dongfack MDJ, Lallemand MC, Kuete V, Mbazoa CD, Wansi JD, Trinh-van-Dufat H, Wandji JMichel S, Wandji J. A new sphingolipid and furanocoumarins with antimicrobial activity from ficus exasperata. Chem Pharm Bull (Tokyo). 2012;60(8):1072-5. doi: 10.1248/cpb.c1200279 , PMID 22863713.

49. Sumi SA, Siraj MA, Hossain A, Mia MS, Afrin S, Rahman MM. Investigation of the key pharmacological activities of ficus racemosa and analysis of its major bioactive polyphenols by HPLC-DAD. Evidence-Based Complementary Alternative Med. 2016;2016:3874516:3874516. doi: $10.1155 / 2016 / 3874516$.

50. Jain R, Rawat S, Jain SC. Phytochemicals and antioxidant evaluation of ficus racemosa root bark. J Pharm Res. 2013;6(6):615-9. doi: 10.1016/j.jopr.2013.06.004.

51. Alqasoumi SI, Basudan OA, Al-Rehaily AJ, Abdel-Kader MS. Phytochemical and pharmacological study of ficus palmata growing in Saudi Arabia. Saudi Pharm J. 2014;22(5):460-71. doi: 10.1016/j.jsps.2013.12.010, PMID 25473335.

52. Kothiyal SC, Saklani S. Isolation and identification of Ficus palmata leaves and their antimicrobial activities. J Sci Res. 2017;9(2):193-200. doi: 10.3329/jsr.v9i2.27806:, 193200PMID 193200.

53. Zhang J, Zhu WF, Xu J, Kitdamrongtham W, Manosroi A, Manosroi J, Feng F. Potential cancer chemopreventive and anticancer constituents from the fruits of Ficus hispida L.f. (Moraceae). J Ethnopharmacol. 2018;214:3746.

54. Mudhafar M. Review of photochemistry for polyalithia longiflia. Discovery. Phytomedicine. 2019;2:33-55.

55. Christian EO, Obumneme OC, Joy O, Edith AN, Okwuchukwu AB, Ngozi NN, Godwin NVO. Comparative phytochemical and nutritional profiles of ficus capensis and cnidoscolus aconitifolius leaves. Int J Res Innovation Appl Sci. 2020;51:16-21.

56. Aboaba SA, Oladosu IA, Ekundayo 0. Chemical composition, acetylcholinesterase inhibitory properties and brine shrimp toxicity of essential oils from Ficus capensis thunb from Nigeria. Int J Biol Chem Sci. 2010;4(5):1494-500. doi: 10.4314/ijbcs.v4i5.65555.

57. Mudhafar M, Zainol I, Jaafar CNA, Alsailawi HA, Desa S. A rreview sstudy on ssynthesis mmethods of agnanoparticles, cconsidering a antibacterial pproperty and ccytotoxicity. Int J Drug Delivery Technol. 2021;2:635-48. 